\title{
CIRED 2019 - Internationale Konferenz über elektrische Verteilnetze
}

\author{
H. Struber OVE
}

Online publiziert am 15. Oktober 2019

(C) Springer-Verlag GmbH Austria, ein Teil von Springer Nature 2019

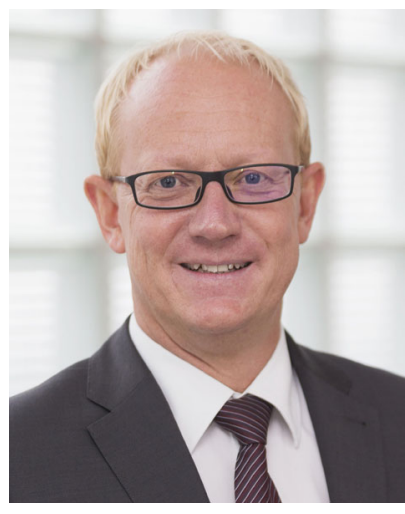

Dipl.-Ing. Herwig Struber MSc
Alle zwei Jahre findet die internationale Konferenz der CIRED über elektrische Verteilnetze statt. Die Konferenz bietet eine ausgezeichnete Plattform zu allen technischen Fragen in Verteilnetzen, dem aktuellen Stand der Technik sowie laufende ForschungsInnovations- und Entwicklungstätigkeit. Neben dem technischen Schwerpunkt umfasst die CIRED den gesamten Bereich der Stromverteilungssysteme und der damit verbundenen Dienst-

leistungen, einschließlich den Fragen der verteilten Stromerzeugung und neuen Entwicklungen.

Die CIRED bildet somit eine exzellente Gelegenheit für all jene, die sich als Teil der Stromverteilungscommunity verstehen, unabhängig davon, ob sie aus dem Verteilungs-, Erzeugungs-, Beratungs-, Dienstleistungs- oder aus dem akademischen Sektor stammen. Nach 2017 in Glasgow fand die 25. CIRED-Konferenz vom 3. bis 6. Juni 2019 in Madrid in Spaniens führendem Konferenzzentrum IFEMA Messe Madrid statt.

Die 25. CIRED-Konferenz 2019 konnte auch einen Rekord an Teilnehmern verzeichnen, 1519 Teilnehmer aus 62 Länder nutzten die vier Tage in Madrid zum umfangreichen Wissens- und Erfahrungsaustausch. Aus Österreich waren 46 Teilnehmer gemeldet. Aus mehr als 1731 zur Konferenz eingereichten Beiträgen wurden 932 Beiträge in einem umfassenden Evaluierungsprozess, in den auch zahlreiche österreichische Experten eingebunden waren, akzeptiert.

Mit 22 österreichischen Beiträgen blieb die Anzahl, verglichen mit den letzten Konferenzen, auf einem sehr hohen Niveau.

Die fachliche Gliederung der Konferenz wurde mit sechs Fachbereichen (Sessions) wie auch schon in den letzten CIRED-Konferenzen aufrechterhalten:

1) Netzkomponenten

2) Spannungsqualität und elektromagnetische Verträglichkeit

3) Betrieb, Steuerung und Schutz

4) Dezentrale Energieerzeugung und aktive Verbrauchsintegration

5) Verteilnetzplanung

6) Geschäftliches Umfeld

Am ersten Konferenztag fanden noch vor der offiziellen Eröffnung sieben Tutorials zu ausgewählten Themen in Verteilnetzen statt. An den folgenden drei Konferenztagen wurden die Beiträge der einzelnen Sessions präsentiert. Dafür standen, aufgrund der sechs Sessions, teilweise zeitgleich abgehaltene Plenum-Vorträge, RoundTable-Diskussionen, geführte Poster-Sessions und Forschungs- und
Innovationsforen statt. Insbesondere die Poster Sessions, bei denen die Autoren ihre Beiträge im direkten Gespräch mit interessierten Experten erläutern konnten, fanden wieder großen Anklang. Die Fachbeiträge wurden von einer umfassenden Herstellerausstellung über verteilnetzrelevante Produkte und Dienstleistungen ergänzt. Im Anschluss an die Konferenz konnten wieder technische Anlagen in der Umgebung von Madrid besichtigt werden. Dieses Jahr wurden die Besichtigung des neuen Iberdrola Trainingscenters sowie eines Hochspannungsprüflabors von LCOE angeboten.

Die österreichische Präsenz bei der CIRED-Konferenz 2019 wurde auch dieses Mal durch eine überproportionale Anzahl von Teilnehmern als auch von Beiträgen unter Beweis gestellt. Dies ist sicherlich auch auf die aktive Rolle der österreichischen Verteilnetzbetreiber, Hersteller, Forschungsinstitute und Universitäten zurückzuführen.

Im Anschluss an die Konferenz wurde die Sitzung des CIRED Directing Committees abgehalten. Wichtigster Punkt war die Weiterentwicklung des erfolgreichen Weges der CIRED, welche nur durch die Unterstützung und Mitarbeit der nationalen CIRED-Komitees möglich war.

Für diejenigen, die an der Konferenz nicht teilnehmen konnten, bietet das österreichische Nationalkomitee wieder zwei Infonachmittage mit Präsentationen über die aktuellen Trends der sechs Sessions an:

CIRED-Infonachmittag des österr. Nationalkomitees

Wien, Dienstag, 28. Jänner 2020

in den Räumlichkeiten des OVE, Eschenbachgasse 9, 1010 Wien

CIRED-Infonachmittag des österr. Nationalkomitees Innsbruck, Dienstag 18. Februar 2020 Hotel Grauer Bär, Universitätsstraße 5-7, 6020 Innsbruck

Die österreichischen CIRED-Veranstaltungen sind auch im OVEVeranstaltungskalender geführt.

Weitere CIRED-Veranstaltungen sind:

CIRED Workshop 2020 - Berlin, Deutschland

4.-5. Juni 2020, Estrel Congress Centre

How to implement flexibility in the distribution system?

CIRED Workshop 2020 - Shanghai, China (1. CIRED

Workshop außerhalb Europas)

26.-27. Oktober 2020

Bringing power to fast growing citites: smart and digital distribution grids

CIRED Conference 2021 - Genf, Schweiz

21.-24. Juni 2021, Messezentrum Palexpo

Struber, Herwig, Salzburg Netz GmbH, Bayerhamerstraße 16, 5020 Salzburg, Österreich (E-Mail: herwig.struber@salzburgnetz.at) 
Die nachfolgenden Beiträge über die CIRED Sessions stellen Kurzfassungen der Inhalte und Trends dar und sollen zu einer intensiveren Befassung mit den Beiträgen der CIRED-Konferenz anregen. Besten Dank auch an die österreichischen Berichterstatter!

Weiterführende Informationen oder Ansprechpartner finden Sie auf den folgenden Websites: http://www.cired.at

http://www.cired.net

http://www.cired2019.org

http://www.cired2020shanghai.org/

https://www.cired2020berlin.org/

http://wwwcired2021.org 